\title{
A new polarized target material : ${ }^{6} \mathbf{L i D}\left({ }^{*}\right)$
}

\author{
A. Abragam, V. Bouffard, Y. Roinel and P. Roubeau \\ DP. H/SRM, CEN Saclay, Orme des Merisiers, B.P. nº 2, 91190 Gif sur Yvette, France \\ (Reçu le 7 mars 1980, acceptéle 16 mai 1980)
}

\begin{abstract}
Résumé. - Des polarisations supérieures à $70 \%$ ont été obtenues dans un échantillon de deutériure de lithium ${ }^{6} \mathrm{LiD}$ pour les spins nucléaires de lithium et de deutérium. Ces résultats font de ${ }^{6} \mathrm{LiD}$ un matériau de cible polarisée dont les performances excèdent considérablement celles de tous les autres matériaux étudiés à ce jour.
\end{abstract}

\begin{abstract}
Polarizations exceeding $70 \%$ have been obtained in a sample of ${ }^{6} \mathrm{LiD}$, for the nuclear spins of lithium and deuterium. These results make ${ }^{6} \mathrm{LiD}$ a polarized target material which outperforms all other materials tried so far.
\end{abstract}

An outstanding problem in the field of polarized targets for elementary particle physics is the production of polarized neutrons. Since free neutrons do not exist in matter (at least at the densities that prevail on earth) one turns to polarized deuterons. The deuteron is a weakly bound structure which can be described with reasonable accuracy as a neutron and a proton with parallel spins and no orbital moment. Organic substances where a large proportion of hydrogen atoms is replaced by deuterium atoms, have been polarized dynamically for that purpose [1] The deuteron polarizations obtained in this way are of the order of $45 \%$, to be compared with proton polarizations in the same samples, of nearly $100 \%$.

The reason for this disparity is well known. It has been proposed [2] and reviewed [3] that for most substances containing more than one nuclear spin species their dynamic polarization results in equal spin temperatures rather than equal polarizations. The latter are related to the common spin temperature by the usual Brillouin functions $B_{I}\left(\gamma_{I} \hbar H_{0} / k_{\mathrm{B}} T_{\mathrm{S}}\right)$ where $T_{\mathrm{S}}=\beta^{-1}$ is the spin temperature in an applied magnetic field $H_{0}$. Thus in lithium hydride ${ }^{7} \mathrm{Li}^{1} \mathrm{H}$ containing a small proportion of ${ }^{6} \mathrm{Li}$, polarizations $P\left({ }^{6} \mathrm{Li}\right) \sim 35 \%$ and $P\left({ }^{7} \mathrm{Li}\right) \sim 80 \%$ were observed simultaneously, in a field $H_{0}=6.5 \mathrm{~T}$ corresponding to a common inverse spin temperature :

$$
\beta=360 \mathrm{~K}^{-1}[3] \text {. }
$$

(*) La version française de cet article a été acceptée aux Comptes Rendus de l'Académie des Sciences et elle est insérée dans le $\mathrm{n}^{\circ}$ du 10. mars 1980.
Larger deuteron polarizations require imperatively lower spin temperatures. It follows from the theory of dynamic nuclear polarization (abbreviated as DNP) that the ultimate nuclear spin temperature reached in a DNP process is an increasing function of the ESR width of the paramagnetic impurities responsible for the DNP [3]. Thus the way to lower nuclear spin temperatures passes through narrower ESR lines and this is where ${ }^{6} \mathrm{LiD}$ comes in.

The paramagnetic impurities used in the dynamic polarization of ${ }^{7} \mathrm{Li}^{1} \mathrm{H}$ are $\mathrm{F}$-centres, produced by fast electron irradiation of the samples [4]. Their line width is determined to a large extent by the hyperfine couplings of the electron of the F-centre with the surrounding ${ }^{6} \mathrm{Li}$ nuclei. The ratio expected on this basis for the rms ESR widths in ${ }^{7} \mathrm{Li}^{1} \mathrm{H}$ and ${ }^{6} \mathrm{LiD}$ is (for unpolarized nuclei) :

$$
\frac{\gamma_{7}}{\gamma_{6}}\left[\frac{I_{7}\left(I_{7}+1\right)}{I_{6}\left(I_{6}+1\right)}\right]^{1 / 2}=3.6
$$

and it is not unreasonable to expect a comparable ratio for the spin-temperatures $T_{\mathrm{S}}\left({ }^{7} \mathrm{Li}{ }^{1} \mathrm{H}\right) / T_{\mathrm{S}}\left({ }^{6} \mathrm{LiD}\right)$. This is to be contrasted with the usual polarized targets materials, where the ESR line width is due mainly to a distribution of electronic $g$-factors and is not modified appreciably by isotopic substitutions of nuclear spins.

Before giving the experimental results obtained in the DNP of ${ }^{6} \mathrm{LiD}$ a further asset of this substance should be mentioned. An important quality criterion of a polarized target material is the ratio $r$ of polarized protons (neutrons) to the total number of protons (neutrons) in the sample, or sometimes in the so- 
called inclusive reactions, the total number of polarized nucleons to the total number of nucleons. Whatever definition we use for $r$, this ratio in ${ }^{6} \mathrm{LiD}$ is 0.25 , if only deuteron polarization is considered. Actually this ratio is 0.5 thanks to the polarization of ${ }^{6} \mathrm{Li}$. ${ }^{6} \mathrm{Li}$ which has the same spin $I=1$, and within $4 \%$ the same gyromagnetic ratio $\gamma_{I}$ as $\mathrm{D}$ has (within $4 \%$ ) the same polarization as D. As far as the nucleon spin polarization is concerned, the ground state ${ }^{6} \mathrm{Li}$ can be described with reasonable accuracy as

$$
{ }^{6} \mathrm{Li}={ }^{2} \mathrm{D}+{ }^{4} \mathrm{He} \text { or } \quad{ }^{6} \mathrm{Li}={ }^{3} \mathrm{He}+{ }^{3} \mathrm{H}
$$

Either scheme yields the magnetic moment of ${ }^{6} \mathrm{Li}$ with an accuracy better than $4 \%$ and either description leads to the value $r=0.5$ for ${ }^{6} \mathrm{LiD}$. It may be pointed out that so far the largest ratio $r$, in substances where large dynamic polarizations have been obtained, occurs in ammonia $\mathrm{NH}_{3}$ where the ratio : polarized protons/all protons is 0.3 and polarized nucleons/all nucleons is 0.176 .

For substances with larger $r$ ratios such as $\mathrm{H}_{2}$ or HD no significant dynamic polarization has been observed so far.

Our results for dynamic polarization in a polycrystalline sample of ${ }^{6} \mathrm{LiD}$, doped with F-centres according to a procedure described in [3] are the following :

$$
H_{0}=6.5 \mathrm{~T} \quad P\left({ }^{6} \mathrm{Li}\right)=71 \% \text {. }
$$

This corresponds to an inverse spin temperature of $825 \mathrm{~K}^{-1}$ that is 2.3 times larger than that currently obtained in ${ }^{7} \mathrm{LiH}$ under comparable conditions.

$$
\begin{array}{ll}
H_{0}=4.8 \mathrm{~T} & P\left({ }^{6} \mathrm{Li}\right)=64 \% \\
H_{0}=2.5 \mathrm{~T} & P\left({ }^{6} \mathrm{Li}\right)=40 \% .
\end{array}
$$

The sample was cooled with a dilution refrigerator. The temperature of the bath was below $200 \mathrm{mK}$ during the DNP. There is no direct means of measuring the temperature of the sample itself, which may be significantly higher. The microwave power dissipated in the cryostat is estimated to be about $100 \mu \mathrm{W}$.

The various values quoted for the magnetic fields correspond to the discrete frequencies of the polarizing microwave sources namely $182 \mathrm{GHz}, 136 \mathrm{GHz}$, $71 \mathrm{GHz}$. The dimensions of the samples were $5 \times 5 \times 0.5 \mathrm{~mm}$ and it is clear that their scaling up to the size currently used for high energy polarized targets would require considerable development work.

The polarization times ranged between 20 and 40 hours which is appreciably longer than in standard target materials and may be inconvenient.

Details of this experiment will be reported elsewhere.

To conclude, we have produced the highest neutron polarization 0.71 ever observed, in a material, ${ }^{6} \mathrm{LiD}$, with the highest ratio of polarized nucleons over all nucleons.

\section{References}

[1] De Boer, W., Borghini, M., Morimoto, K., Ninikoski, T. O. and F. UDo, J. Low Temp. Phys. 15 (1974) 249.

[2] Buishvili, L. L., Sov. Phys. JETP 22 (1966) 1277.

Borghini, M., Phys. Rev. Lett. 20 (1968) 419.
[3] Abragam, A. and Goldman, M., Rep. Prog. Phys. 41 (1978) 395.

[4] Roinel, Y., Bouffard, V. and Roubeau, P., J. Physique 39 (1978) 1097 